Article

\title{
Design and Optimization of Three-Phase Dual-Active-Bridge Converters for Electric Vehicle Charging Stations
}

\author{
Duy-Dinh Nguyen ${ }^{1,2, *(\mathbb{D})}$, Ngoc-Tam Bui ${ }^{2,3}{ }^{\mathbb{D}}$ and Kazuto Yukita ${ }^{1}$ \\ 1 Aichi Institute of Technology, Toyota 470-0356, Japan; yukita@aitech.ac.jp \\ 2 Hanoi University of Science and Technology, Hanoi 100803, Vietnam; tambn@shibaura-it.ac.jp \\ 3 Shibaura Institute of Technology, Tokyo 135-8548, Japan \\ * Correspondence: nguyenduydinh@aitech.ac.jp; Tel.: +81-0565-48-8121
}

Received: 27 November 2019; Accepted: 25 December 2019; Published: 27 December 2019

check for updates

\begin{abstract}
In this paper, design and optimization method of a three-phase dual-active-bridge DC/DC converter is discussed. Three single phase transformers connected in star-star configuration were designed with large leakage inductance aiming to eliminate the need for external inductors. Switching frequency, peak flux density, number of turns, number of layers, etc., were optimized using non-linear programming technique for minimizing the overall converter loss. Experimental results on a $10 \mathrm{~kW}$ prototype show that the optimized converter can operate efficiently an efficacy of up to $98.65 \%$ and a low-temperature rise of less than 70 degrees Celsius on both transformers and semiconductor devices.
\end{abstract}

Keywords: three-phase dual-active-bridge converter; nonlinear programming; inequality constraints; genetic algorithm; inductor-integrated transformer

\section{Introduction}

Electric vehicles (EVs) play an importance role in reducing $\mathrm{CO}_{2}$ emission, as they are powered by chemical batteries or super capacitors, rather than by fuel combustion. Recently, some new ideas have been proposed to make use of EVs more effectively. Among those, the idea of using EVs as power system stabilizers via vehicle-to-grid (V2G) or vehicle-to-home (V2H) technologies is very interesting and promising thanks to their significant storage capabilities [1,2]. Accordingly, the EV would be connected to the utility via a so-called EV supply equipment (EVSE) and a charging outlet (such as Chademo, CCS/Combo, etc.) when it is not in used in a quite long time. Depending on the actual needs of the grid, the EV could be charged or discharged to help stabilize the grid. In order for the energy stabilizer mode to function, the EVSE must be a bidirectional converter that allows the power to transfer bidirectionally. An example of such the system is depicted in Figure 1.

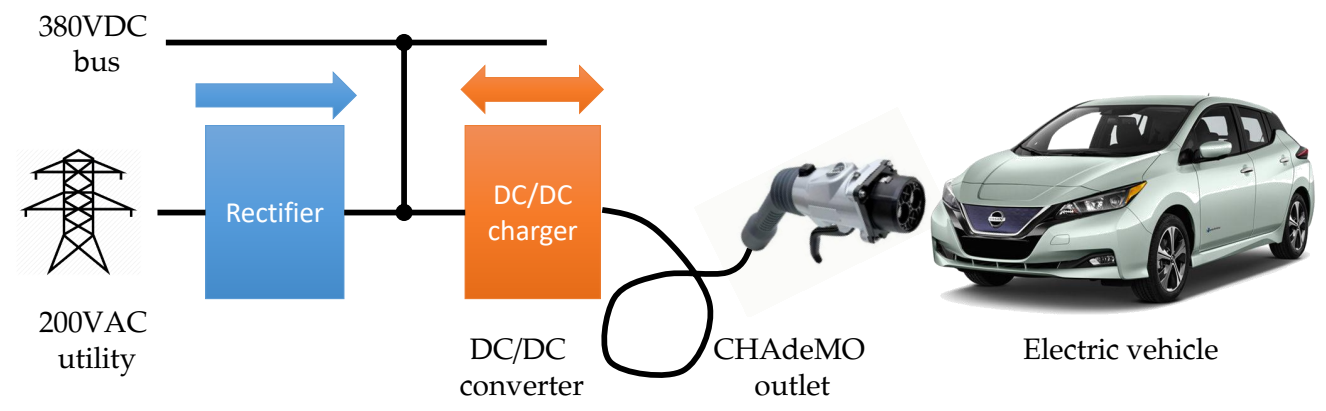

Figure 1. Three-phase dual active bridge converter topology. 
The EVSE in Figure 1 consists of two parts: a unidirectional rectifier and a bidirectional DC/DC charger. The output of the rectifier is tied to a $380 \mathrm{~V}$ DC bus of a DC micro-grid, from which the bidirectional DC/DC converter charges the electric vehicle via a CHAdeMO outlet. When the vehicle is left unused, the outlet remains connected for charging or for reversing the power back to the DC bus. Thanks to that, the DC micro-grid can be stabilized. This paper focuses on designing and optimization of the bidirectional DC/DC converter of such an EVSE (from now on, the DC charger).

From another aspect, according to [3], high frequency current ripple causes negative effect on the battery lifetime. Therefore, it is necessary to design a DC charger with low output current ripple. It should also have high power density, high efficiency, be galvanic isolated, and be bidirectional. Among many DC/DC converter topologies, dual-active-bridge (DAB) has been found to be the most appropriate choice for this application [4-6].

There are two popular types of DAB converter: single-phase and three-phase, both having inherent soft-switching, bidirectional, and isolation characteristics. Unlike the single-phase counter part, the three-phase structure can triple the effective frequency and reduce the amplitude of the ripple. Hence, input and output filters can be downsized; the transformer RMS current is also lower and power density is higher than that of the single-phase version. Up till now, numerous studies on three-phase $\mathrm{DAB}$ converter have been published [6-10] regarding various topics, including modulation and control to system design and optimization, etc. While many discussions on designing and optimizing the single-phase DAB can be found in the literature, only a few are available for three-phase topology.

Of course, the design and optimization procedures that are applied for single-phase DAB converter can be applicable for three-phase DAB. For example, the typical approach is starting from loss modeling, formulating the optimization problem, and then solving the problem by using some techniques, such as that reported in [11]. However, in [11], an external inductor was used, and it is preferable to integrate the inductor into the transformer for better space saving and more efficient performance. Moreover, the switching frequency was fixed in that research; only the number of turns and the inductance were optimized. The conduction loss model used in that research did not address the dependency of AC resistance of winding on the proximity effect, which varies correspondingly to the number of turns and layers [12].

In [13], the inductor-integrated transformer in a single-phase DAB converter was designed and optimized by using the particle swarm optimization method. The skin and proximity effects were regarded in loss modeling. However, conduction loss and switching loss of semiconductor devices were not considered. Therefore, the overall converter system was not optimized; only transformers were. The research reported in [14] modeled both electronics loss and transformer loss. The transformer used shell-type winding for better integration of leakage inductance. However, only the control variables (phase shift, switching frequency) were optimized. The transformer structure was fixed and not optimized.

Design and optimization of three-phase DAB converter for DC charging applications are resolved in this paper. Both transformer structure and operation parameters are optimized. Inductors are integrated into the transformers for better space and cost saving. The value of leakage inductance, number of turns, number of layers, etc., are also optimized. For better accuracy, skin and proximity effects are also addressed in modeling the copper loss of transformers. A nonlinear programming technique was employed to find the optimal parameter set. Due to certain facts, including that the window area of the transformer is limited, the semiconductor devices have a certain switching capability, the operating temperature needs to be handled as well, etc., some inequality constraints were considered for the optimization problems. Afterward, the optimized transformer was verified by a finite element method magnetic (FEMM) analysis and by an experimental study.

This paper is actually an improved version of that reported in [15]. The converter was modeled with consideration that number of turns and number of layers are integers. That makes the optimizing function discontinuous and non-differentiable. Therefore, a nonlinear programming method based on gradient, such as the Newton-Raphson, becomes inapplicable. Besides, in order to find the global 
optima, the Global Optimization Toolbox of MATLAB \& Simulink from Mathworks incorporation was employed. Furthermore, operation in the whole power range was examined in this improved version. The paper is structured as follows: analysis in the steady state is presented in Section 2; transformer design method is described in Section 3; the loss model can be found in Section 4; the optimization algorithm is narrated in Section 5; the optimization, simulation, and experimental results are provided in Section 6; and finally, some conclusions are given in Section 7.

\section{Steady State Analysis}

Figure 2 describes the circuit diagram of a three-phase dual-active-bridge (from now on, DAB3) converter. Three single phase high-frequency transformers are employed for easier fabrication and implementation of the system. It assumes that all transformers have the same leakage inductance of $L_{k}$ and the same winding ratio of $n: 1$. According to [8], among some transformer configurations, the $\mathrm{Y}-\mathrm{Y}$ topology requires smaller leakage inductance to send a specific amount of power. That also means a smaller number of turns of transformer winding is required, resulting in smaller winding resistance. Therefore, in this study, the $\mathrm{Y}-\mathrm{Y}$ connection is used aiming for performance optimization. Notes here that, delta-type transformer structures can be transformed into star connection by using the following transformation:

$$
L_{Y a}=\frac{L_{D b} L_{D c}}{L_{D a}+L_{D b}+L_{D c}} .
$$

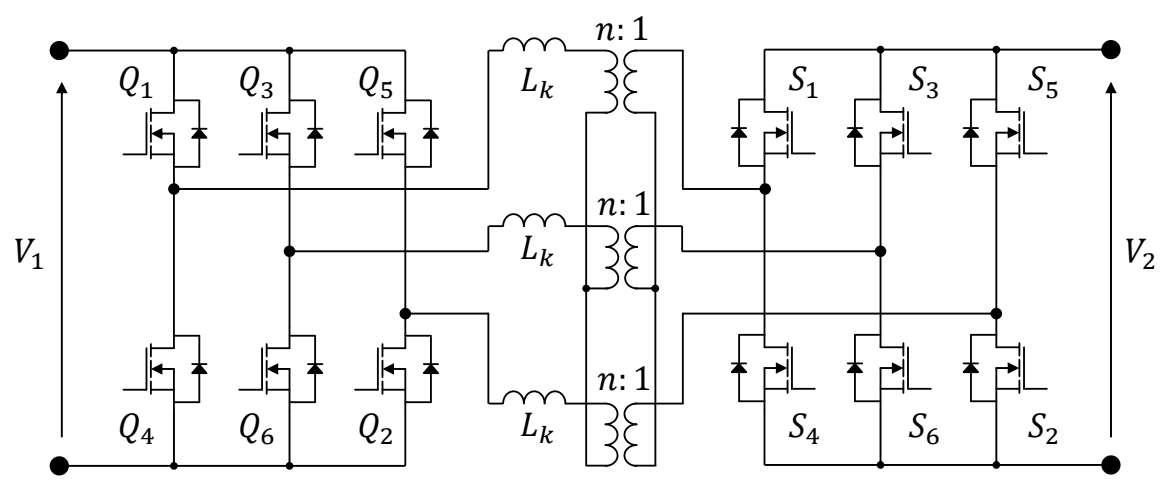

Figure 2. Three-phase dual active bridge converter topology.

Two three-phase inverters are located at two sides of the transformers. The conventional phase shift modulation and 180-degree-conduction-mode are used to control the converter. The switching frequency is $f_{s}$ which needs to be optimized. Voltages at the two DC terminals are $V_{1}$ and $V_{2}$, respectively. The voltages across the transformer winding have the four-level form and are $\psi$ degrees shifted from each other as shown in Figure 3, where $\psi$ is the phase shift control angle.

Assuming that the phase shift $\psi$ is smaller than $\frac{\pi}{3}$ for better reactive power reduction, according to [8], the transition current $I_{0}$ to $I_{5}$ at the steady state can be calculated by (1):

$$
\left\{\begin{array}{l}
I_{0}=-I_{M}\left(2(1-M)+\frac{3 M \psi}{\pi}\right) \\
I_{1}=I_{M}\left(-2(1-M)+\frac{3 \psi}{\pi}\right) \\
I_{2}=I_{M}\left(-(1-M)+\frac{3 M \psi}{\pi}\right) \\
I_{3}=I_{M}\left(-(1-M)+\frac{6 \psi}{\pi}\right) \\
I_{4}=I_{M}\left((1-M)+\frac{6 M \psi}{\pi}\right) \\
I_{5}=I_{M}\left((1-M)+\frac{3 \psi}{\pi}\right)
\end{array}\right.
$$


where $I_{M}=\frac{V_{1}}{18 f_{s} L_{k}} ; M$ is the voltage conversion ratio, $M=\frac{n V_{2}}{V_{1}}$; and $\psi$ is the phase shift, $\psi<\frac{\pi}{3}$.

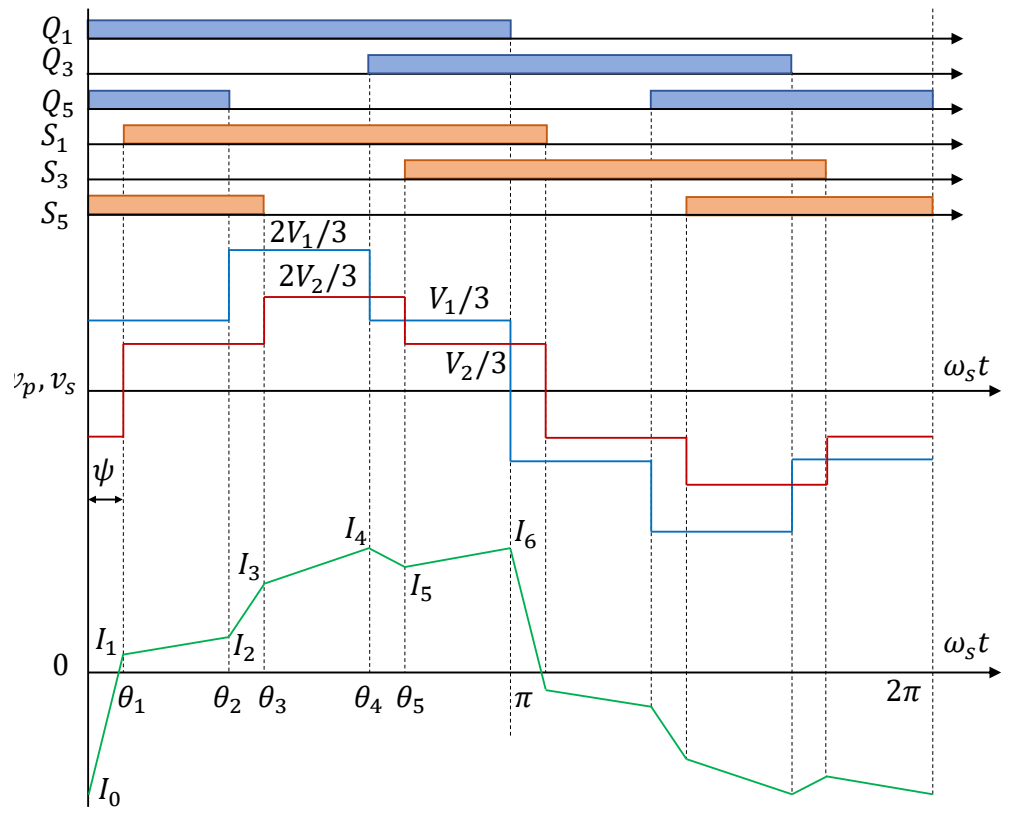

Figure 3. Theoretical voltage and current waveforms.

The root-mean-squared (rms) transformer current, rms switch currents, peak flux density (obtained by calculating the volt-second product of each conducting interval and assuming a balanced volt-second distribution of transformers), and output power are calculated by equations from (2) to (6) as follows:

$$
\begin{aligned}
I_{p r i, r m s} & =\frac{I_{M} \sqrt{3}}{3} \sqrt{5(1-M)^{2}+27\left(2-\frac{\psi}{\pi}\right)\left(\frac{\psi}{\pi}\right)^{2} M} \\
I_{Q x, r m s} & =\frac{I_{p r i, r m s}}{\sqrt{2}} \\
I_{S x, r m s} & =\frac{I_{Q x, r m s}}{n} \\
B_{p k} & =\frac{V_{1}}{18 f_{s} N_{1} A_{c}}\left(1+M\left(1-\frac{3 \psi}{2 \pi}\right)\right) \\
P_{\text {out }} & =\frac{3 M V_{1} I_{M}}{2} \psi\left(4-\frac{3 \psi}{\pi}\right),
\end{aligned}
$$

where $N_{1}$ is the number of turns of the primary winding; $A_{c}$ is the cross section area of the center limb of the magnetic core, assuming that $\mathrm{E}$ core is used.

\section{Transformer Design}

At the first step, some preliminary parameters must be determined:

- Maximum output power;

- Nominal input and output voltages;

- Current density $\left(J=6-10 \mathrm{~A} / \mathrm{mm}^{2}\right)$;

- Window utilization factor $\left(K_{u}=0.2-0.4\right.$ for transformer);

- Initial switching frequency and flux density. 
After that, from (2), (5) and (6), the core size and winding wire size can be selected according to Ap or $\mathrm{Kg}$ methods [16]. Here, in order to reduce the skin and proximity effects, Litz wire is preferable. Three transformers with the traditional core-typed winding structure, as depicted in Figure 4, were designed. There are only two windings in each transformer, primary and secondary; no interleaving. The centralized wounding technique was used. The insulation between two windings was made thick for leakage inductance integration purpose. The insulation thickness is, thus, a variable for tuning the leakage inductance.

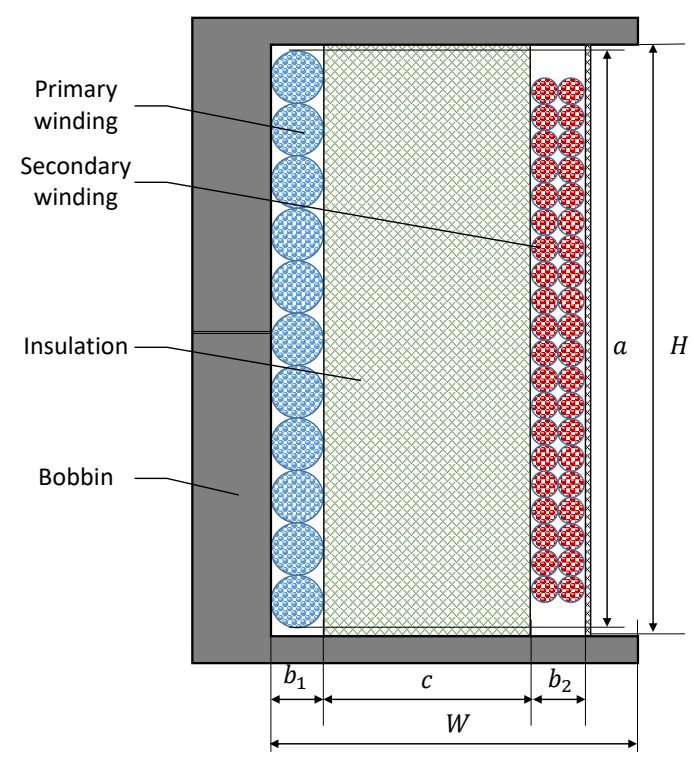

Figure 4. Transformer with thick insulation configuration.

According to [16], the leakage inductance can be calculated by:

$$
L_{k}=\frac{\mu_{0}(M L T) N_{1}^{2}}{a}\left(c+\frac{b_{1}+b_{2}}{3}\right)
$$

where $\mu_{0}$ is the permeability of vacuum, $\mu_{0}=4 \pi \times 10^{-7} ; M L T$ is the mean-length-turn of the core; $a$ is the winding height; $b_{1}$ and $b_{2}$ are the windings' thicknesses; $c$ is the insulation thickness; and $N_{1}$ is the number of turns of primary winding, from (5). $N_{1}$ can be derived as (8):

$$
N_{1}=\operatorname{ceil}\left(\frac{V_{1}}{18 f_{s} B_{p k} A_{c}}\left(1+M\left(1-\frac{3 \psi}{2 \pi}\right)\right)\right)
$$

where ceil() is the ceiling (round-up) function.

Now, let $m_{1}$ and $m_{2}$ be the number of layers of primary and secondary windings; the winding dimension will be:

$$
\begin{aligned}
a & =O D_{1} \times \frac{N_{1}}{m_{1}} \\
b_{1}+b_{2} & =O D_{1} m_{1}+O D_{2} m_{2}
\end{aligned}
$$

where $O D_{1}$ and $O D_{2}$ are the outer dimensions of winding wires. Let $H$ be the window height of the bobbin. The winding height $a$ must be smaller than $H$. From (9), we get:

$$
N_{1} \leq \frac{H}{O D_{1}} m_{1} .
$$


Due to the round shape of the wire and the necessary space for creepage and clearance distance, the whole core height cannot be used for winding. Therefore, by denoting $k_{c_{1}}$ as the utilization factor of the core height, $k_{c_{1}} \leq 0.9, m_{1}$ and $m_{2}$ can be determined by:

$$
\begin{aligned}
& m_{1}=\operatorname{ceil}\left(\frac{N_{1} O D_{1}}{k_{c_{1}} H}\right) \\
& m_{2}=\operatorname{ceil}\left(\frac{N_{2} O D_{2}}{k_{c_{1}} H}\right) .
\end{aligned}
$$

Since $b_{1}$ and $b_{2}$ depend on the chosen current density $J$, and have less effect on leakage inductance value, as formulated in (7), the tuning parameter for $L_{k}$ is the insulation thickness $c$. Note that the summation $\left(b_{1}+b_{2}+c\right)$ must be smaller than the total window width $W$ of the bobbin. The leakage inductance is highest at $c=c_{\max }$ :

$$
c \leq c_{\max }=W-\left(b_{1}+b_{2}\right) .
$$

From (7)-(14), the maximum achievable leakage inductance for a given core is determined by:

$$
L_{k} \leq L_{k, \max }=\frac{\mu_{0}(M L T) H}{O D_{1}} m_{1}^{2}\left(\frac{W}{O D_{1}}-\frac{2}{3}\left(m_{1}+\frac{O D_{2}}{O D_{1}} m_{2}\right)\right) .
$$

The leakage inductance is minimum when there is no insulation between the two windings $(c=0)$ :

$$
L_{k} \geq L_{k, \min }=\frac{\mu_{0}(M L T) N_{1} m_{1}}{3}\left(m_{1}+\frac{O D_{2}}{O D_{1}} m_{2}\right) .
$$

From (6), for a given output power of $P_{m}$, the maximum phase shift is calculated by:

$$
\psi_{m}=\frac{2 \pi}{3}\left(1-\sqrt{1-\frac{f_{s}}{f_{s, \max }}}\right),
$$

where $f_{s, \text { max }}=\frac{M V_{1}^{2}}{9 L_{k} P_{m}}$. Obviously, in order for (17) to be valid, the switching frequency must be less than the maximum value of $f_{s, \max }$ :

$$
f_{s} \leq f_{s, \max }=\frac{M V_{1}^{2}}{9 L_{k} P_{m}}
$$

From (8), (17) and (18), we have:

$$
f_{s} \geq \frac{V_{1}}{18 N_{1} A_{c} B_{p k}}\left(1+M \sqrt{1-\frac{f_{s}}{f_{s, \max }}}\right) .
$$

Solving (19) for $f_{s}$, the lower boundary of switching frequency is:

$$
f_{s} \geq f_{s, \min }=\lambda f_{s, \max }\left(1-\frac{\lambda}{2} M^{2}+M \sqrt{1-\lambda+\frac{\lambda^{2}}{4} M^{2}}\right),
$$

where $\lambda=\frac{P}{2 M V_{1}} \times \frac{L_{k}}{N_{1} A_{c} B_{p k}}$. 


\section{Power Loss Modeling}

\subsection{Power Electronics Loss}

Assuming that zero-voltage transition is achieved, turn-on loss can thus be ignored. The overall power dissipation on the Silicon-Carbide Metal-Oxide-Semiconductor Field-Effect Transistor (SiC-MOSFETs) can be estimated by:

$$
\Delta P_{p e}=\Delta P_{\text {cond }}+\Delta P_{\text {sw }}
$$

where $\Delta P_{\text {cond }}$ and $\Delta P_{s w}$ are conduction loss and turn-off loss respectively:

$$
\begin{aligned}
\Delta P_{\text {cond }} & =6 R_{d s, O N}\left(I_{Q x, r m s}^{2}+n^{2} I_{S x, r m s}^{2}\right) \\
\Delta P_{s w} & =3 V_{1}\left(I_{0}+M I_{1}\right) t_{f} f_{s}
\end{aligned}
$$

with $R_{d s, O N}$ and $t_{f}$ being the ON resistance and the falling time of the MOSFET; both are given in the device data-sheet; $I_{0}$ and $I_{1}$ are the transition currents given in (1); $I_{Q x, r m s}$ and $I_{S x, r m s}$ are the rms currents flowing through primary and secondary switches, which are given in (3) and (4), respectively.

\subsection{Transformer Loss}

Transformer loss includes copper loss dissipating on windings and ferrite loss on the magnetic core as follows:

$$
\Delta P_{t r}=\Delta P_{C u}+\Delta P_{F e},
$$

where $\Delta P_{C u}$ and $\Delta P_{F e}$ are the winding loss (or copper loss) and core loss (ferrite loss). Using the Steimetz equation, the core loss can be estimated by:

$$
\Delta P_{F e}=3 k_{c} f_{s}^{\alpha} B_{p k}^{\beta} g
$$

where $B_{p k}$ is the peak flux density, and $g$ is the weight of two core halves. $k_{c}, a$ and $b$ are the Steimetz coefficients: $k_{c}=0.00004855, \alpha=1.62$, and $\beta=2.63$ [16].

The copper loss is determined by (24):

$$
\Delta P_{C u}=3 R_{a c} I_{p r i, r m s}^{2}
$$

where $R_{a c}$ is the primary-referred AC resistance. Note that the AC resistance depends strongly on the switching frequency and the winding geometry by the skin and proximity effects. According to [12], the $\mathrm{AC}$ resistance can be estimated for Litz wire as follows:

$$
R_{a c}=R_{d c} A_{s t r}\left(\frac{\sinh 2 A_{s t r}+\sin 2 A_{s t r}}{\cosh 2 A_{s t r}-\cos 2 A_{s t r}}+\frac{2}{3}\left(m^{2} n_{s t r}-1\right) \frac{\sinh A_{s t r}-\sin A_{s t r}}{\cosh A_{s t r}+\cos A_{s t r}}\right),
$$

where $A_{s t r}=\frac{1}{\delta}\left(\frac{\pi}{4}\right)^{0.75} \sqrt{\frac{d_{b}^{3}}{d_{w}}} ; \delta$ is the skin depth, $\delta=\frac{6.62 \times 10^{-2}}{\sqrt{f_{s}}} ; d_{b}$ and $d_{w}$ are the bare and outer diameters of a single strand of the Litz wire; $m$ is the number of layers; $n_{s t r}$ is the number of strands; $R_{d c}$ is the DC resistance of the winding:

$$
R_{d c}=(M L T) N \frac{r_{s t r}}{n_{s t r}}
$$

where $N$ is the number of turns (i.e., $N_{1}$ for primary winding and $N_{2}$ for secondary winding); $r_{s t r}$ is the resistance density of a strand; and MLT is the mean-length-turn of the magnetic core. 


\subsection{Capacitor Loss}

Since there is ripple in the input and output currents of the converter, the ripple will be filtered out by the input and output capacitors and dissipate some power on the series resistance of the capacitors. The capacitor loss is determined by:

$$
\Delta P_{\text {cap }}=r_{\text {cin }} I_{\text {cin }, r m s}^{2}+r_{\text {cout }} I_{\text {cout }, r m s}^{2}
$$

where $r_{\text {cin }}$ and $r_{\text {cout }}$ are the series resistances of capacitors; $I_{\text {cin,rms }}$ and $I_{\text {cout,rms }}$ are the input and output rms capacitor currents which can be easily derived from (1).

Finally, the total power dissipation of the converter is derived as:

$$
\Delta P_{\text {tot }}=\Delta P_{p e}+\Delta P_{t r}+\Delta P_{\text {cap }}
$$

\section{Optimization}

In order to maximize system efficiency, the overall power dissipation should be minimized. The analysis above shows that the four most basic parameters that affect all others are the switching frequency $f_{s}$, the peak flux density $B_{p k}$, the voltage conversion ratio $M$, and the leakage inductance $L_{k}$. When $f_{s}, B_{p k}, M$, and $L_{k}$ are known, all other parameters can be easily derived.

Here, some additional constraints are considered. First, after designing the transformer, according to [16], one can estimate the temperature rise of the core by (28).

$$
\Delta T_{t r}=450\left(\frac{\Delta P_{t r}}{3 A_{t}}\right)^{0.826}
$$

where $A_{t}$ is the total surface of the transformer; $A_{t}$ can be calculated without difficulty when the core dimensions are known.

The temperature rise of power electronics devices can also be estimated if the thermal resistance of the heatsink $R_{h s}$ is given. Assuming that the heat generated from semiconductor devices is evenly distributed on the heatsink, then the temperature rise is:

$$
\Delta T_{p e}=\Delta P_{p e} R_{h s}
$$

The maximum temperature rise should not be greater than a certain allowable value of $\Delta T_{\max }$.

$$
\max \left\{\Delta T_{t r}, \Delta T_{p e}\right\} \geq \Delta T_{\max }
$$

It is also worth noticing that all analyses above are conducted under the assumption that soft-switching is achieved. According to [8] and considering the required deadtime $T_{d}$ in modulation, the lowest soft-switching boundary when $\psi<\pi / 3$ is:

$$
\psi \geq \psi_{\min }=\frac{2 \pi}{3}(1-M)+2 \pi f_{s} T_{d}
$$

At that operational point, the transferred power is:

$$
P_{\text {min }}=\frac{M V_{1}^{2}}{12 f_{s} L_{k}} \psi_{\min }\left(4-\frac{3}{\pi} \psi_{\min }\right) \text {. }
$$
as (32):

The rated power $P$ should be greater than $P_{\min }$ at least $k_{p}$ times for a soft-switching range of $k_{p}: 1$

$$
P \geq k_{p} P_{\text {min }}
$$

Eventually, the optimization problem is defined as follows: 


\section{*) Minimization problem:}

Find a set of $\left(f_{s}, B_{p k}, L_{k}, M\right)$ to minimize the total converter loss:

$$
f\left(f_{s}, B_{p k}, L_{k}, M\right)=\Delta P_{t o t} \rightarrow \min
$$

subject to:

$$
\left\{\begin{array}{l}
g_{1}\left(f_{s}, B_{p k}, L_{k}, M\right)=F_{s, \min }-f_{s} \leq 0 \\
g_{2}\left(f_{s}, B_{p k}, L_{k}, M\right)=f_{s}-F_{s, \max } \leq 0 \\
g_{3}\left(f_{s}, B_{p k}, L_{k}, M\right)=L_{k}-L_{k, \max } \leq 0 \\
g_{4}\left(f_{s}, B_{p k}, L_{k}, M\right)=L_{k, \min }-L_{k} \leq 0 \\
g_{5}\left(f_{s}, B_{p k}, L_{k}, M\right)=\max \left\{\Delta T_{t r}, \Delta T_{p e}\right\}-\Delta T_{\max } \leq 0 \\
g_{6}\left(f_{s}, B_{p k}, L_{k}, M\right)=k_{p} P_{\min }-P \leq 0,
\end{array}\right.
$$

where $k_{p}: 1$ is the power range of the converter, $k_{p}$ should be as great as possible. In this paper, $k_{p}$ is chosen as 5 for a power range of 5:1.

\section{*) Solution:}

In order to solve the above optimization problem, there are numerous of available methods. However, note here that the optimizing function is non-continuous and non-differentiable, as it includes a round-up (ceiling) function. Therefore, explicit methods based on gradient are not applicable; instead, heuristic techniques such as the genetic algorithm (GA), differential evolution (DE), particle swarm optimization (PSO), etc., are preferred [17]. In this paper, GA is selected due to its simplicity and feasibility.

In terms of implementation, among many available tools, the Global Optimization Toolbox (GOT) [18] of MATLAB and Simulink appears to be a very good choice, as it can generate the global optima rather than a local one. Besides, its usage is also very simple and does not require deep knowledge on the fitness function. Therefore, the GA function of GOT was employed to solve the above optimization problem. The fitness function was given by (33). The nonlinear inequalities constraints were (34). There were no linear and/or equality constraints. Flow chart diagram of the whole optimization and design process is given in Figure 5.

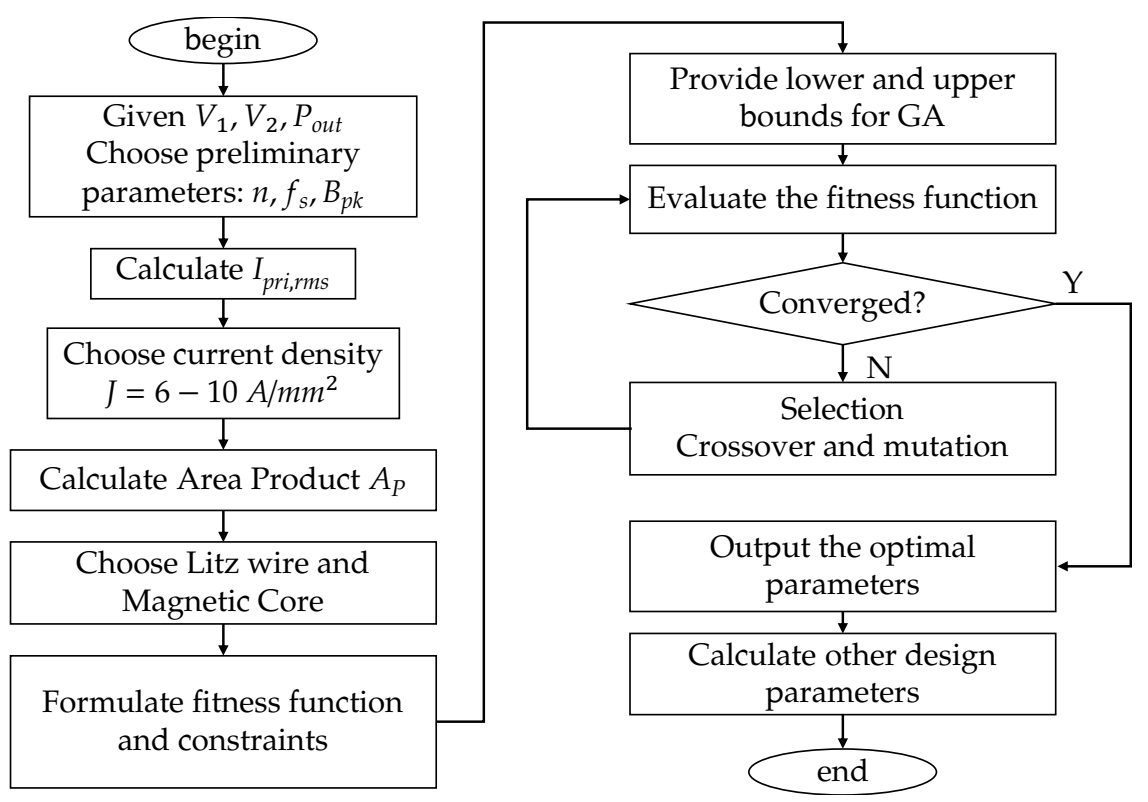

Figure 5. Design and optimization flow chart. 


\section{Results and Discussion}

\subsection{Optimization Results}

The proposed design and optimization strategy was applied to design a $10 \mathrm{~kW}$, three-phase dual-active-bridge (DAB) converter for battery charging application. From (2), the primary current can be calculated with a preliminary phase shift angle. Afterward, the current density $J$ is chosen to be, for example, $9 \mathrm{~A} / \mathrm{mm}^{2}$. Then, the magnetic core of ETD54/28/19 from TDK corporation is selected. The specification and preliminary parameters of the required converter are given in Table 1.

Table 1. Specification and preliminary parameters.

\begin{tabular}{ccc}
\hline Parameter & Symbol & Value \\
\hline Input voltage & $V_{1}$ & $380 \mathrm{~V}$ \\
Output voltage & $V_{2}$ & $240-480 \mathrm{~V}$ \\
Nominal output voltage & $V_{2, n o m}$ & $380 \mathrm{~V}$ \\
Rated power & $P$ & $10 \mathrm{~kW}$ \\
\hline Maximum temperature rise & $\Delta T$ & $70^{\circ} \mathrm{C}$ \\
Preliminary frequency & $f_{s}$ & $50 \mathrm{kHz}$ \\
Preliminary phase shift & $\psi$ & $30 \mathrm{degrees}$ \\
Preliminary peak flux density & $B_{p k}$ & $250 \mathrm{mT}$ \\
Calculated inductance & $L_{k}$ & $15.56 \mu \mathrm{H}$ \\
Desired current density & $J$ & $9 \mathrm{~A} / \mathrm{mm}^{2}$ \\
\hline Selected wire & & Litz 1050AWG44 \\
Selected ferrite core & & ETD54/28/19 (N87) \\
SiC MOSFET & & CREE C2M0040120D \\
\hline
\end{tabular}

Lower and upper bounds of optimizing variables are given in Table 2. Running the above optimization algorithm, optimization progress is shown in Figure 6. The optimal loss is 210.02 $\mathrm{W}$, achieved at the switching frequency of $72.71 \mathrm{kHz}$, the peak flux density of $137.6 \mathrm{mT}$, the leakage inductance of $5.05 \mathrm{mT}$, and the voltage conversion ratio of 1:1. The best efficiency was obtained as $97.9 \%$ at the rated power of $10 \mathrm{~kW}$. The temperature rise of transformer was calculated as 70 degrees-just right—equal to the maximum allowable value given in Table 1. Other parameters are listed in Table 3.

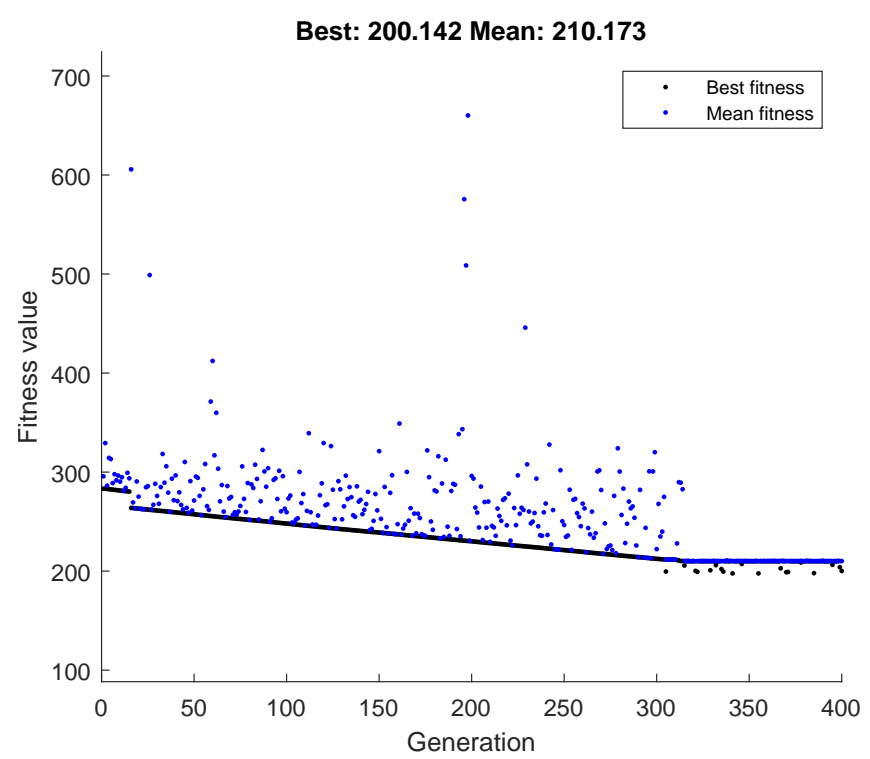

Figure 6. Optimization process using genetic algorithm. 
Table 2. Lower and upper bounds for genetic algorithm.

\begin{tabular}{cccc}
\hline Parameter & Symbol & Lower Bound & Upper Bound \\
\hline Switching frequency & $f_{s}$ & $50 \mathrm{kHz}$ & $300 \mathrm{kHz}$ \\
Peak flux density & $B_{p} k$ & $50 \mathrm{mT}$ & $250 \mathrm{mT}$ \\
Leakage inductance & $L_{k}$ & $1 \mu \mathrm{H}$ & $10 \mu \mathrm{H}$ \\
Voltage conversion ratio & $M$ & 0.5 & 2 \\
\hline
\end{tabular}

Since $72.71 \mathrm{kHz}$ is not suitable for programming, it was round-up to $75 \mathrm{kHz}$. This causes a slightly deviation in the total loss and efficiency of the converter compared to the optimal value. However, that deviation is insignificant and can be ignored. The modified optimal parameters and all other design parameters are described in Table 3.

Also listed in Table 3 is the competing parameter set. For the same transformer design, we operated the system at a different switching frequency (and thus, different phase shift), and then compared the performance to confirm whether or not the optimized parameters are better. It was not possible to lower switching frequency, or the control range constraint would have been violated. Therefore, the competing switching frequency was $100 \mathrm{kHz}$.

Table 3. Optimal and modified parameters.

\begin{tabular}{cccccc}
\hline Parameter & Symbol & Optimized Value & Modified Value & Competing Value & Unit \\
\hline Switching frequency & $f_{s}$ & 72.71 & 75 & 100 & $\mathrm{kHz}$ \\
Peak flux density & $B_{p k}$ & 137.6 & 133.13 & 97.68 & $\mathrm{mT}$ \\
Leakage inductance & $L_{k}$ & 5.05 & 5.05 & 5.05 & $\mu \mathrm{H}$ \\
Conversion ratio & $M$ & 1 & 1 & 1 & \\
Number of turns & $N_{1}: N_{2}$ & $15: 15$ & $15: 15$ & $15: 15$ & \\
Phase shift angle & $\psi$ & 13.1 & 13.54 & 18.46 & degrees \\
AC resistance & $R_{A C}$ & 31.86 & 31.99 & 33.61 & $\mathrm{~m} \Omega$ \\
Transformer temperature rise & $\Delta T_{t r}$ & 70.0 & 69.75 & 69.15 & ${ }^{\circ} \mathrm{C}$ \\
Heatsink temperature rise & $\Delta T_{p e}$ & 40.7 & 41.44 & 49.73 & ${ }^{\circ} \mathrm{C}$ \\
Power electronics loss & $\Delta P_{p e}$ & 162.80 & 165.76 & 198.93 & $\mathrm{~W}$ \\
Transformer loss & $\Delta P_{t r}$ & 42.97 & 42.78 & 42.34 & $\mathrm{~W}$ \\
Total loss & $\Delta P_{t o t}$ & 210.02 & 212.94 & 247.24 & $\mathrm{~W}$ \\
Efficiency@P $P_{\text {rated }}$ & $\eta$ & $97.9 \%$ & $97.87 \%$ & $97.53 \%$ & \\
\hline
\end{tabular}

Figure 7 illustrates the calculated efficiency and temperature curves obtained by the optimized and competing parameters using the model in the previous sections when varying the phase shift from the minimum value to the nominal one. As shown in Figure 7a, the minimum power was calculated at the minimum phase shift, which is equivalent to the dead-time period. Maximum efficiency of near $98.5 \%$ was achieved at around $4 \mathrm{~kW}$. The expected efficiency at the rated power was $97.87 \%$, as mentioned above. The plotted temperature curve indicates the trend of the greater value between temperature of transformers and power electronics devices with the assumption that the ambient temperature is 15 degree Celsius. At the rated power, the hottest spot was about 85 degree Celsius. Note that this calculation assumes there is no forced air cooling for both transformers and semiconductors, only passive cooling. The characteristics are same when considering the competing parameter set. However, the efficiency is expected to be slightly lower while temperature rise is a little bit less than that when applying the optimized parameter set (due to smaller transformer loss), as can be seen in Figure 7b. 


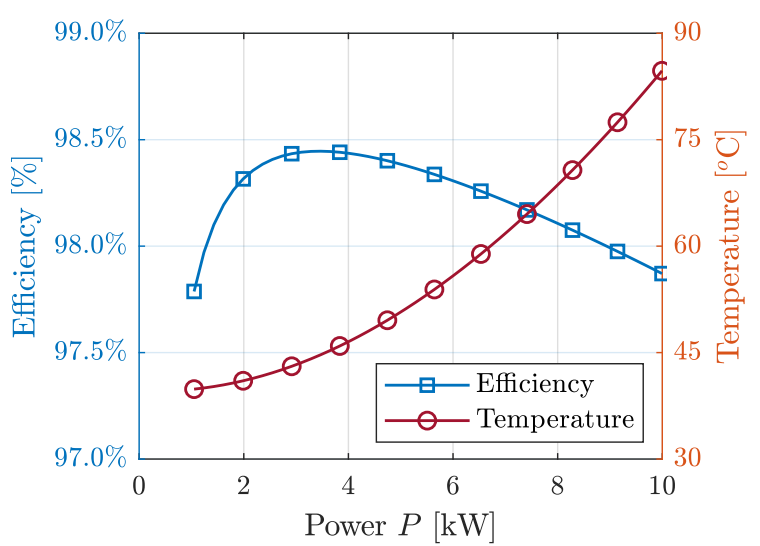

(a)

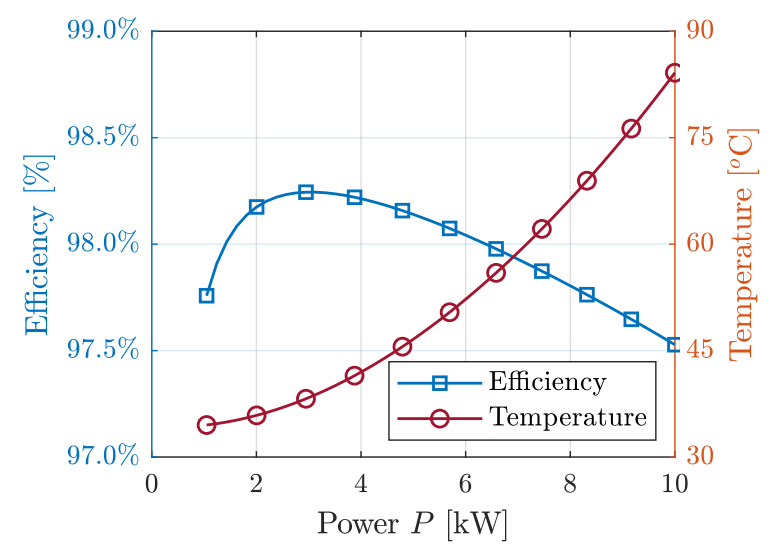

(b)

Figure 7. Calculated efficiency and temperature obtained by: (a) optimized parameters; (b) competing parameters.

\subsection{Simulation and Experimental Results}

Before conducting experiments, a finite-element analysis (FEA) using the Finite-Element-Methodmagnetic (FEMM) version 4.2 [19] was carried out to validate the designed transformers. The flux density obtained by the FEA is illustrated in Figure 8. The flux has higher density at the two outer limbs then at the center one. The peak flux density is $150 \mathrm{mT}$, which is $12.5 \%$ greater than the estimation value. Except for that, other parameters including leakage inductance, AC resistance, and copper and ferrite losses are almost identical to the designed values. Therefore, it is reasonable to fabricate three transformers, namely, $Y_{1}, Y_{2}$, and $Y_{3}$, according to the designed parameters. The complete prototype of the converter is shown in Figure 9.

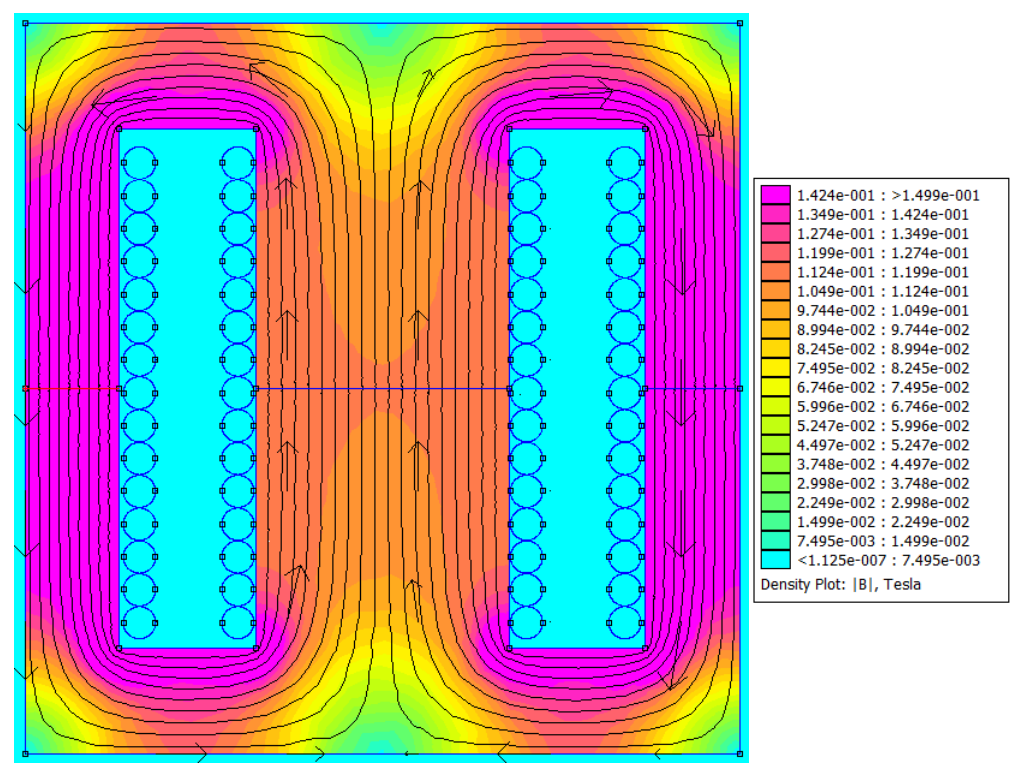

Figure 8. Finite-element-analysis using FEMM 4.2. 


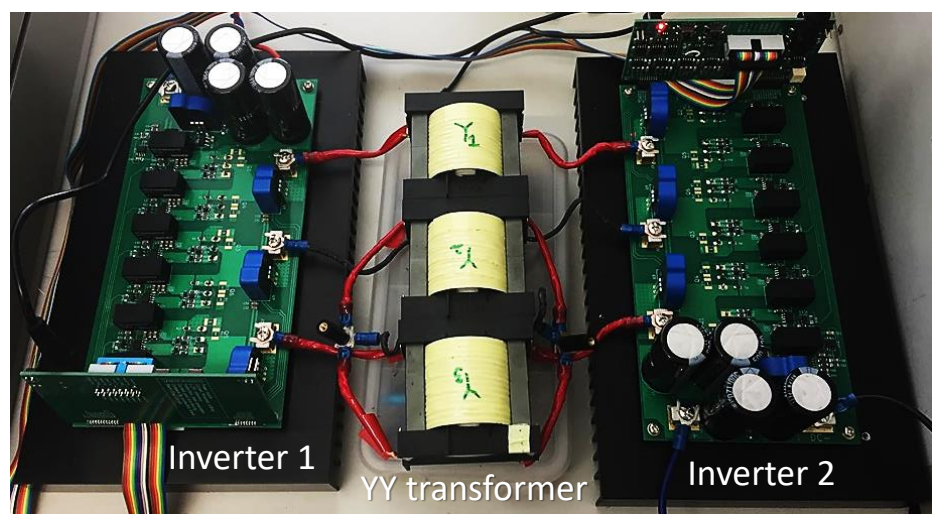

Figure 9. A $10 \mathrm{~kW}$, three-phase DAB converter prototype.

A detailed comparison between calculations, Finite-element analysis (FEA) results, and measurements is given in Table 4. It can be seen that measurements matched calculation and FEA results very well. The simulated leakage inductance was $4.87 \mu \mathrm{H}$, while the expected value was $5.05 \mu \mathrm{H}$, only $3.5 \%$ different. The prediction of transformer loss was also very close to the FEA simulation results. However, the simulated peak flux density was about 13\% higher than expected. That is because the FEMM simulation is 2D, so two outer limbs of the core are treated as rectangular (while in fact, two outer limbs of the ETD core do not have rectangular shapes), making the effective cross-section area $\left(A_{c}\right)$ slightly smaller than the real parameter. On the other hand, the center limb which has cylinder shape in deed is also treated as rectangular, and thus, the flux density in the center limb in FEA simulation is smaller than expected. Anyhow, the difference of peak flux density contributes only to the difference on core loss, which is not significant in this design. Since the transformer designed can be confirmed by FEA simulation, three single phase transformers were built accordingly. However, there are small differences between the actual transformers. This distinction may lead to unevenly loss and heat distributions among phases. It is also worth noting that the measured AC resistances were slightly greater than expected due to the non-ideal winding arrangement. In reality, the actual transformer losses would be slightly higher than estimated. Nevertheless, it can be confirmed here that the converter models and optimization strategy presented in the previous sections are reasonable.

Table 4. Design validation.

\begin{tabular}{|c|c|c|c|c|c|c|c|}
\hline \multirow{2}{*}{ Parameter } & \multirow{2}{*}{ Symbol } & \multirow{2}{*}{ Calculation } & \multirow{2}{*}{ FEA } & \multicolumn{3}{|c|}{ Measurement } & \multirow{2}{*}{ Unit } \\
\hline & & & & $Y_{1}$ & $Y_{2}$ & $Y_{3}$ & \\
\hline Leakage inductance & $L_{k}$ & 5.05 & 4.87 & 4.92 & 4.9 & 4.82 & $\mu \mathrm{H}$ \\
\hline Magnetizing inductance & $L_{m}^{n}$ & 1.2 & 1.17 & 1.33 & 1.13 & 1.24 & $\mathrm{mH}$ \\
\hline AC resistance @ $f_{s, o p t}$ & $R_{A C}$ & 31.99 & 30.81 & 36.5 & 36.5 & 35.2 & $\mathrm{~m} \Omega$ \\
\hline Peak flux density & $B_{p k}$ & 133.13 & 149.9 & - & - & - & $\mathrm{mT}$ \\
\hline Copper loss & $\Delta P_{C u}$ & 10.8057 & 10.19 & - & - & - & $\mathrm{W}$ \\
\hline Core loss & $\Delta P_{F e}$ & 3.4555 & 3.03 & - & - & - & W \\
\hline
\end{tabular}

As the transformers were validated, two inverters made of SiC MOSFET C2M0040120D from CREE incorporation were placed at primary and secondary sides. A TMS320F28335 control card is employed to generate pulse-width-modulation (PWM) signals. A programmable power supply connects to the input side of Inverter 1, whereas a DC electronic load connects to the DC side of Inverter 2. The experiment system is depicted in Figure 10. 


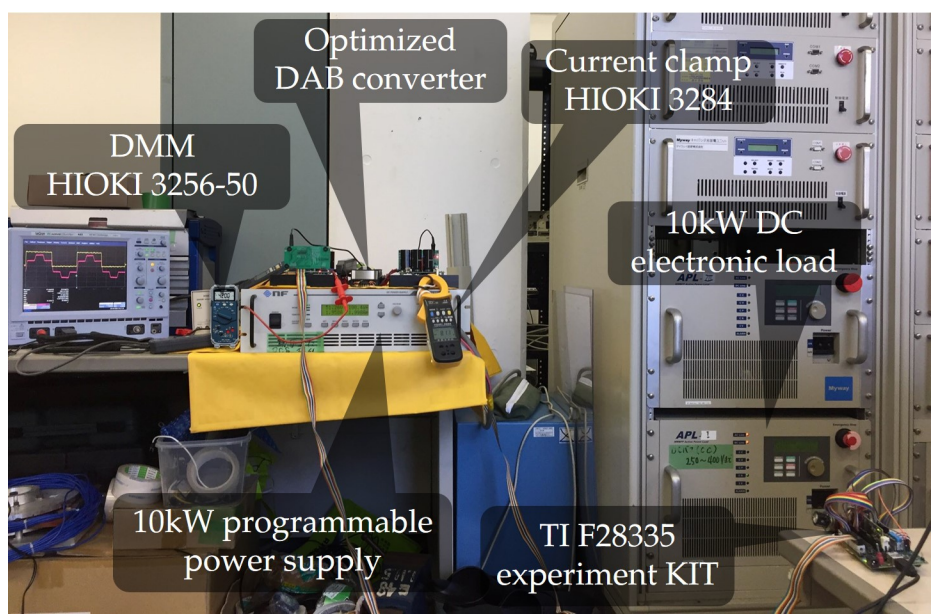

Figure 10. A $10 \mathrm{~kW}$, three-phase DAB converter prototype.

Dead-time was fixed at $100 \mathrm{~ns}$ (equivalent to about 2.7 electrical degrees). The input voltage was set at $380 \mathrm{~V}$. The DC electronics load was configured to operate in the constant resistance mode whose resistance was changed in an open-loop manner to keep the input and output voltage equal. The phase shift was then increased gradually from $\psi_{\min }$ until reaching the maximum power. At each step, efficiency and temperature were recorded. Drain-source voltage of a primary switch and phase current waveform at $9.3 \mathrm{~kW}$ are demonstrated in Figure 11. As shown, soft-switching was achieved; both waveforms are smooth as there are no ringings or spikes.

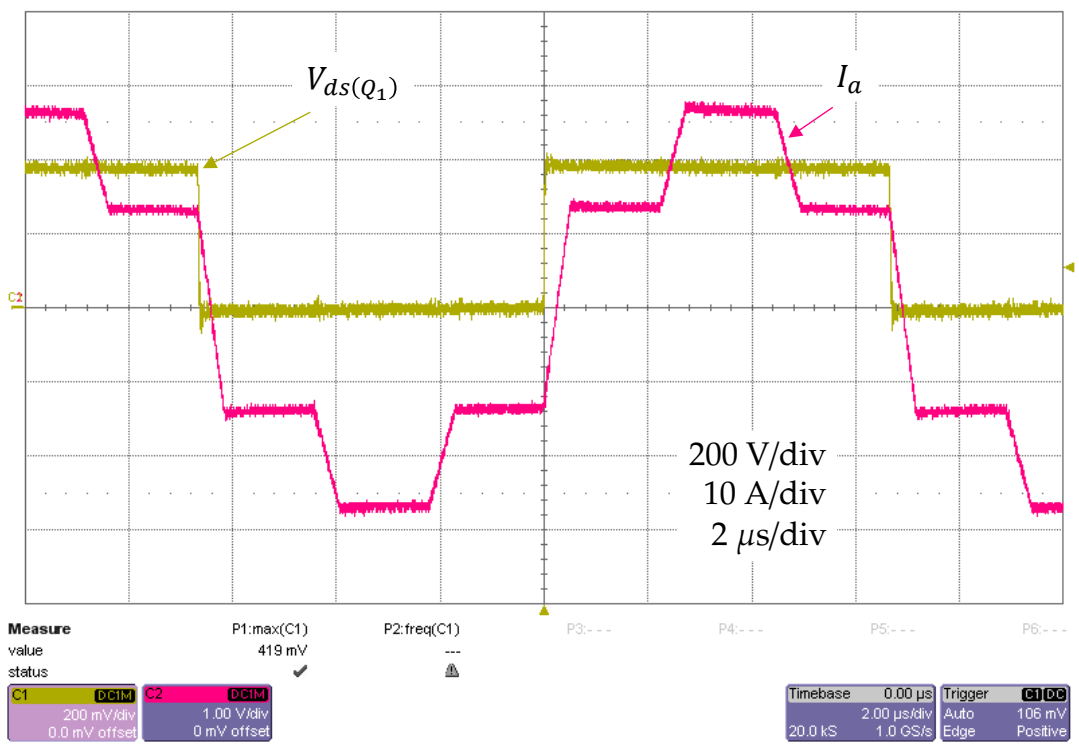

Figure 11. Voltage and current waveforms at $9.3 \mathrm{~kW}$.

Efficiency in the whole power range at unity voltage conversion ratio when using the optimized parameter set is shown in Figure 12. Due to the current limitation of the power supply, the maximum power that can be investigated is only $9.3 \mathrm{~kW}$. The maximum efficiency of $98.65 \%$ is obtained at around $3.6 \mathrm{~kW}$, and the average efficiency over the whole power range is $97.91 \%$, as shown in Figure 12a. The measured results match well with the calculations in the low and mid-power ranges. When the power increases, the converter performance is, however, not as good as expected. For example, the estimated efficiency at $9.3 \mathrm{~kW}$ is $97.9 \%$ but the measured result shows only $97.15 \%$; in other words, there is a $0.75 \%$ error in the determination of the efficiency. The reason may be due to the increment of drain-source resistance of MOSFETs when temperature changes, which was not regarded in the loss model above. Another reason is the mismatch of actual parameters of the real, hand-made 
transformers when compared to the ideal values. Voltage drops on cables connecting input and output of the converter to the power supply and load also contribute to the error between modeling and experiment. Note that at $10 \mathrm{~kW}$, a $1 \mathrm{~V}$ drop on one cable may lead to a $0.25 \%$ reduction in the overall efficiency.

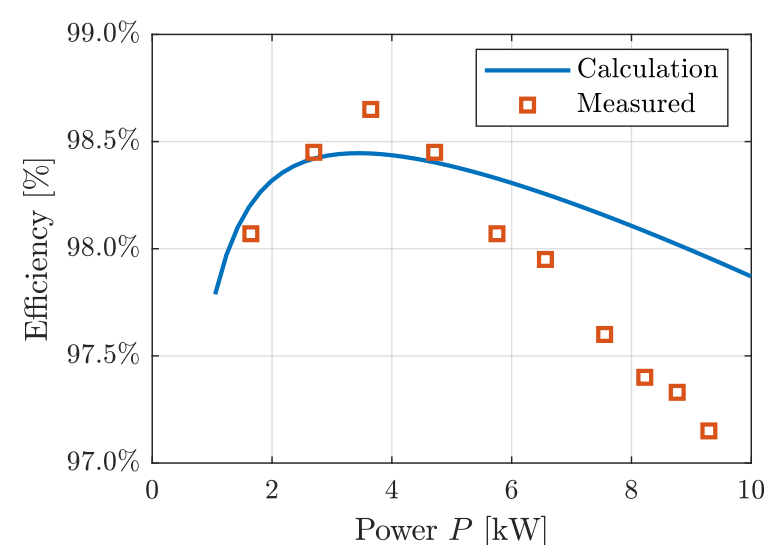

(a)

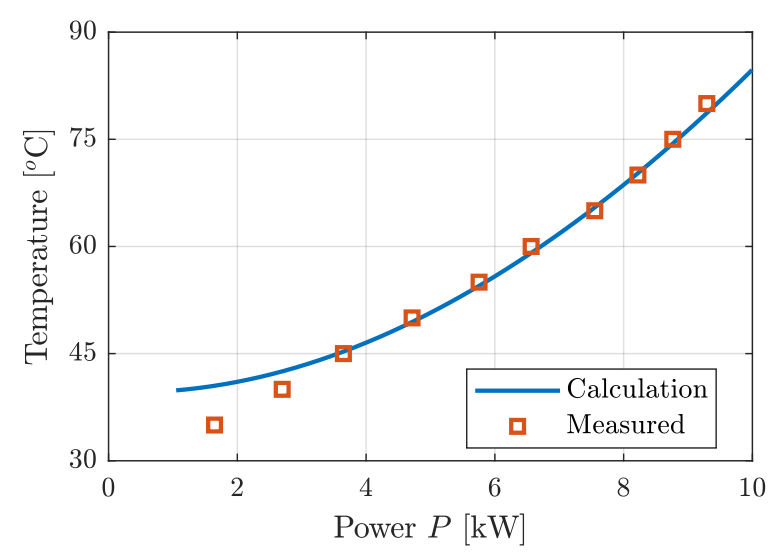

(c)

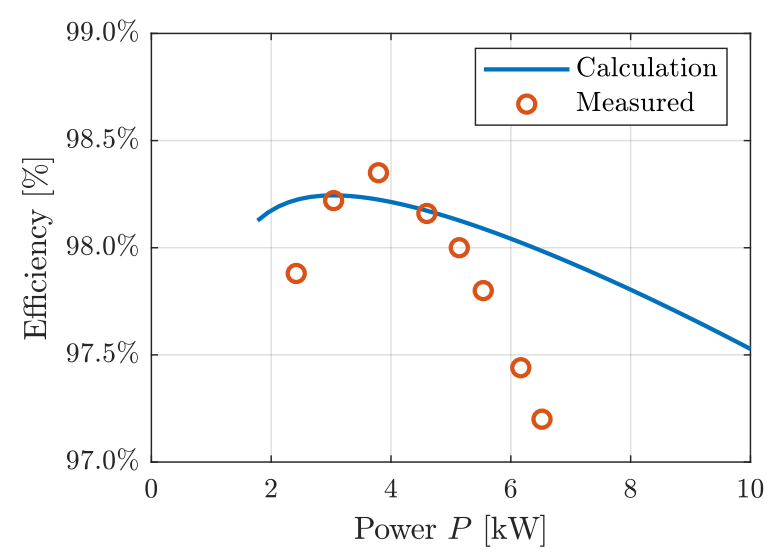

(b)

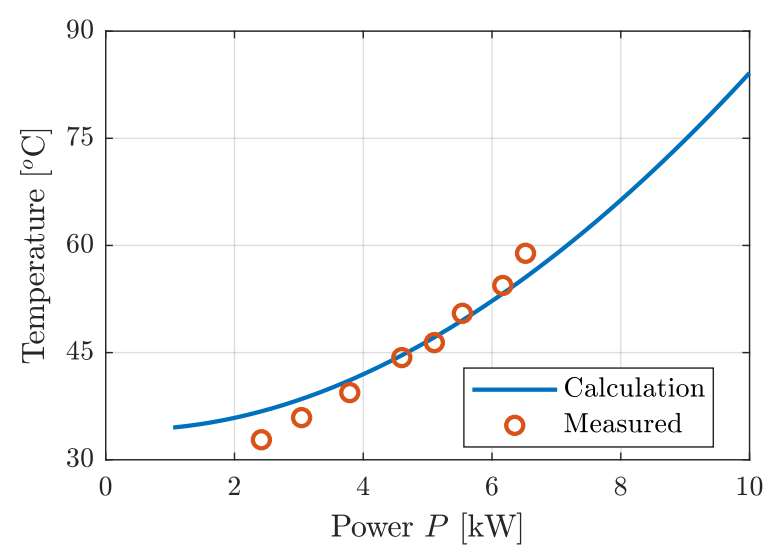

(d)

Figure 12. Performance in the soft-switching range. (a) Efficiency when using the optimal parameter set; (b) efficiency when using the competing parameter set; (c) temperature when using the optimal parameter set; (d) temperature when using the competing parameter set.

The measured temperature also matches well with calculation in the mid and high-power range, as can be seen in Figure 12c. In the low-power range, the temperature was overestimated in calculations, as the measurement values were about 5 degrees cooler than expected. Notes here that the plotted temperatures are the hottest spots on the converter regardless of transformers or inverters being measured. And note also that there is no forced air cooling on either heatsinks or transformers, only passive cooling. For example, as illustrated in Figure 13, the temperature measurement at $8.2 \mathrm{~kW}$ using a FLIR camera shows that the hot spots on the transformer and inverter were 71 and 63 degrees Celsius, respectively, whereas the expected value was 70.7 degrees. These results indicate that the converter loss and heat model, and the optimized strategy, are reliable. Furthermore, if the converter has forced-air cooling, it can perform even better.

It is also worth paying attention to Figure 13b. The loss appears to not be distributed evenly among three legs. The temperature of leg $\mathrm{c}$ of the secondary inverter seems to be hotter than the others. That is comprehensible, since the three transformers are not identical, as described in Table 4. A slight difference in transformer parameters leads to uneven loss and heat distribution, as shown in Figure 13b. In order to avoid this phenomenon, better transformer fabrication is preferable. An alternative method 
is to modify the modulation strategy for evenly current distribution between phases. However, this topic is beyond the scope of this paper.

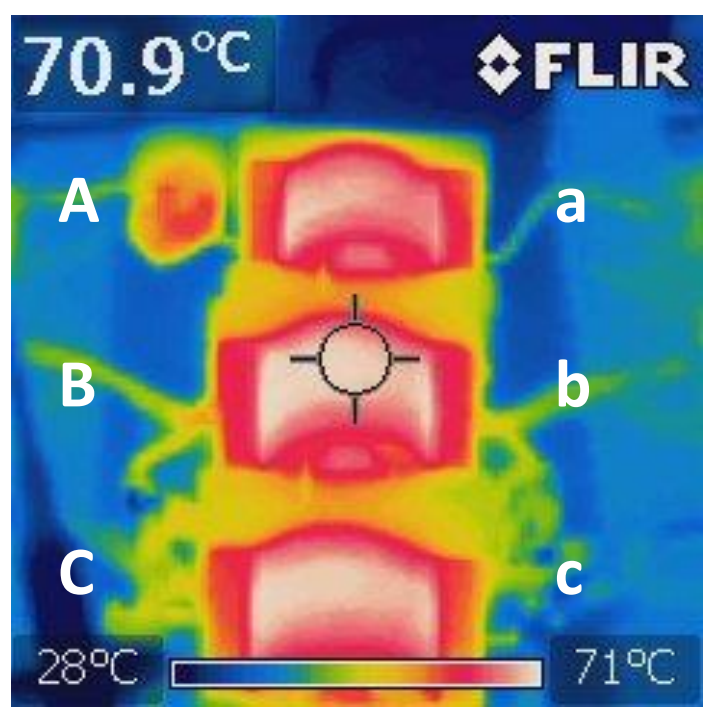

(a)

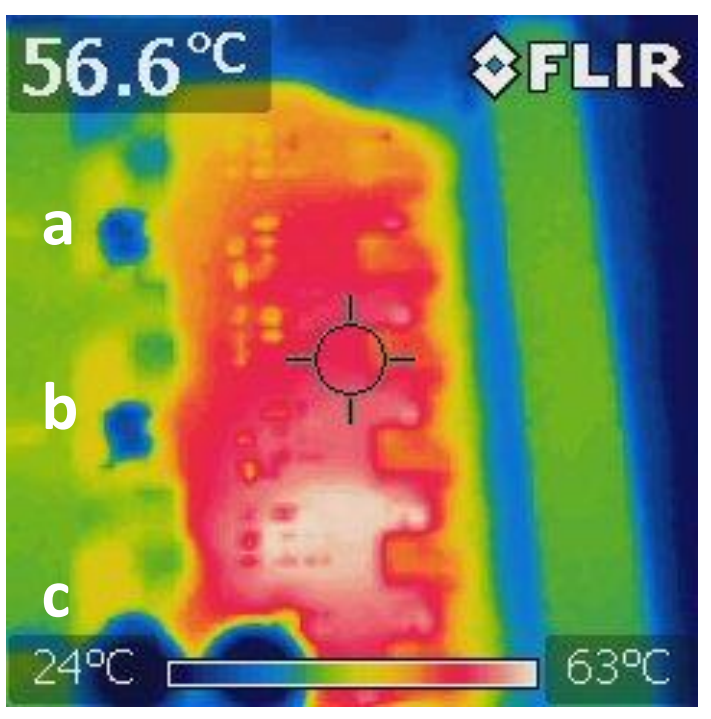

(b)

Figure 13. Temperature at $9.3 \mathrm{~kW}$ : (a) transformer and (b) secondary inverter.

As expected, when using the competing parameter set, the efficiency was not as high as that when switching the converter at the optimized frequency (see Figure 12b). The best performance was only $98.3 \%$, around $4 \mathrm{~kW}$. When increasing the power, the efficiency drops rapidly. At $6.5 \mathrm{~kW}$, the converter can transfer only $97.2 \%$ of the input power, while that value is $97.9 \%$ when using the optimized switching frequency. However, the system is cooler, as predicted above, when the measured temperature at $6.5 \mathrm{~kW}$ is about 58.9 degrees Celsius compared to 60 degrees of the previous case (Figure 12d). Hence, in terms of efficacy, it can be concluded that the converter with the optimized parameters performs better.

\section{Conclusions}

This paper presented the detail and accurate loss modeling for three-phase, dual-active-bridge DC/DC converters with inductor-integrated transformers. After that, an optimization strategy was proposed to minimize the converter loss. Several nonlinear inequality constraints were regarded. There are four variables that were optimized, including switching frequency, peak flux density, leakage inductance, and the voltage conversion ratio. Due to the highly nonlinear optimizing function, the genetic algorithm was employed to optimize the converter. Finite-element-analysis showed that the converter model is reliable. Experimental results confirmed that the optimized design of the converter had the maximum efficiency of $98.65 \%$ and average efficiency in the power range of $97.91 \%$.

Author Contributions: D.-D.N. proposed the main idea, built and performed experiments; N.-T.B. designed the Genetic Algorithm; K.Y. provides supporting ideas, comments and funding; all members contributed significantly in writing and revising the paper. All authors have read and agreed to the published version of the manuscript.

Funding: Knowledge base Aichi Research and development base that supports high value-added manufacturing-Project ID: PI6.

Conflicts of Interest: The authors declare no conflict of interest. 


\section{References}

1. Kempton, W.; Tomić, J. Vehicle-to-grid power implementation: From stabilizing the grid to supporting large-scale renewable energy. J. Power Sources 2005, 144, 280-294. [CrossRef]

2. Ota, Y.; Taniguchi, H.; Nakajima, T.; Liyanage, K.M.; Baba, J.; Yokoyama, A. Autonomous distributed V2G (vehicle-to-grid) satisfying scheduled charging. IEEE Trans. Smart Grid 2011, 3, 559-564. [CrossRef]

3. Uddin, K.; Moore, A.D.; Barai, A.; Marco, J. The effects of high frequency current ripple on electric vehicle battery performance. Appl. Energy 2016, 178, 142-154. [CrossRef]

4. He, P.; Khaligh, A. Comprehensive analyses and comparison of $1 \mathrm{~kW}$ isolated DC-DC converters for bidirectional EV charging systems. IEEE Trans. Transp. Electrif. 2016, 3, 147-156. [CrossRef]

5. Zhu, L.; Taylor, A.R.; Liu, G.; Bai, K. A Multiple-Phase-Shift Control for a SiC-Based EV Charger to Optimize the Light-Load Efficiency, Current Stress, and Power Quality. IEEE J. Emerg. Sel. Top. Power Electron. 2018, 6, 2262-2272. [CrossRef]

6. Haghbin, S.; Alatalo, M.; Yazdani, F.; Thiringer, T.; Karlsson, R. The design and construction of transformers for a $50 \mathrm{~kW}$ three-phase dual active bridge dc/dc converter. In Proceedings of the 2017 IEEE Vehicle Power and Propulsion Conference (VPPC), Belfort, France, 11-14 December 2017; pp. 1-5.

7. van Hoek, H.; Neubert, M.; De Doncker, R.W. Enhanced modulation strategy for a three-phase dual active bridge-Boosting efficiency of an electric vehicle converter. IEEE Trans. Power Electron. 2013, 28, 5499-5507. [CrossRef]

8. Baars, N.H.; Everts, J.; Wijnands, C.G.; Lomonova, E.A. Performance evaluation of a three-phase dual active bridge dc-dc converter with different transformer winding configurations. IEEE Trans. Power Electron. 2016, 31, 6814-6823. [CrossRef]

9. Segaran, D.; Holmes, D.; McGrath, B. Comparative analysis of single-and three-phase dual active bridge bidirectional dc-dc converters. Aust. J. Electr. Electron. Eng. 2009, 6, 329-337. [CrossRef]

10. Mainali, K.; Tripathi, A.; Madhusoodhanan, S.; Kadavelugu, A.; Patel, D.; Hazra, S.; Hatua, K.; Bhattacharya, S. A Transformerless Intelligent Power Substation: A three-phase SST enabled by a $15-\mathrm{kV} \mathrm{SiC}$ IGBT. IEEE Power Electron. Mag. 2015, 2, 31-43. [CrossRef]

11. Iyer, V.M.; Gulur, S.; Bhattacharya, S. Optimal design methodology for dual active bridge converter under wide voltage variation. In Proceedings of the 2017 IEEE Transportation Electrification Conference and Expo (ITEC), Chicago, IL, USA, 22-24 June 2017; pp. 413-420.

12. Wojda, R.; Kazimierczuk, M. Winding resistance of litz-wire and multi-strand inductors. IET Power Electron. 2012, 5, 257-268. [CrossRef]

13. Qin, H.; Kimball, J.W.; Venayagamoorthy, G.K. Particle swarm optimization of high-frequency transformer. In Proceedings of the IECON 2010-36th Annual Conference on IEEE Industrial Electronics Society, Glendale, AZ, USA, 7-10 November 2010; pp. 2914-2919.

14. Jauch, F.; Biela, J. Generalized modeling and optimization of a bidirectional dual active bridge DC-DC converter including frequency variation. IEEJ J. Ind. Appl. 2015, 4, 593-601. [CrossRef]

15. Nguyen, D.D.; Kazuto, Y.; Katou, A.; Yoshida, S. Design optimization of a three-phase Dual-Active-Bridge converter for charging stations. In IEEE Vehicle Power and Propulsion Conference-VPPC 2019; IEEE: Hanoi, Vietnam, 2019; in process.

16. McLyman, C.W.T. Transformer and Inductor Design Handbook; CRC Press: Boca Raton, FL, USA, 2016.

17. Kachitvichyanukul, V. Comparison of three evolutionary algorithms: GA, PSO, and DE. Ind. Eng. Manag. Syst. 2012, 11, 215-223. [CrossRef]

18. Global Optimization Toolbox. Available online: https://www.mathworks.com/products/globaloptimization.html (accessed on 20 November 2019) .

19. Finite Element Method Magnetics 4.2. Available online: http://www.femm.info/wiki/HomePage (accessed on 20 November 2019) .

(C) 2019 by the authors. Licensee MDPI, Basel, Switzerland. This article is an open access article distributed under the terms and conditions of the Creative Commons Attribution (CC BY) license (http:/ / creativecommons.org/licenses/by/4.0/). 